Cipango $\begin{aligned} & \text { Cipango } \\ & \text { Cahiers d'études japonaises }\end{aligned}$

19 | 2012

Le Japon et le fait colonial II

\title{
Les traces du colonialisme dans la littérature japonaise contemporaine
}

\section{Minato Kawamura}

Traducteur : Anne Bayard-Sakai

\section{OpenEdition \\ Journals}

Édition électronique

URL : https://journals.openedition.org/cipango/1712

DOI : 10.4000/cipango.1712

ISSN : 2260-7706

Éditeur

INALCO

Édition imprimée

Date de publication : 30 octobre 2012

ISBN : 978-2-85831-204-7

ISSN : $1164-5857$

Référence électronique

Minato Kawamura, "Les traces du colonialisme dans la littérature japonaise contemporaine », Cipango [En ligne], 19 | 2012, mis en ligne le 31 mars 2014, consulté le 30 juin 2021. URL : http:// journals.openedition.org/cipango/1712 ; DOI : https://doi.org/10.4000/cipango.1712

Ce document a été généré automatiquement le 30 juin 2021.

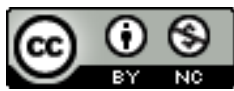

Cipango est mis à disposition selon les termes de la Licence Creative Commons Attribution - Pas d'Utilisation Commerciale 4.0 International. 


\title{
Les traces du colonialisme dans la littérature japonaise contemporaine
}

\author{
Minato Kawamura \\ Traduction : Anne Bayard-Sakai
}

1 Après la reddition sans condition des armées japonaises en août 1945, le Japon est entré dans l'après-guerre. Chacun sait que cette période correspond à celle appelée « aprèslibération » en Corée et à Taiwan, libération par rapport à une situation de colonisé par l'Empire du Grand Japon (Dai nippon teikoku 大日本帝國). Autrement dit, l'aprèsguerre japonais équivaut à cette période d'après-libération pour les territoires jusqu'alors colonisés, Taiwan et la péninsule coréenne (et bien sûr également la Mandchourie, la Micronésie et Sakhaline). C'est pourquoi, la littérature de l'aprèsguerre peut également être appelée littérature de la période de l'après-libération, ou encore de l'époque postcoloniale.

2 Or dans le Japon de l'après-guerre, l'idée que la littérature de l'après-guerre (cette littérature qui a duré un long moment et qui, à bien des égards, perdure encore aujourd'hui) est une littérature postcoloniale est demeurée peu remarquée. Bien entendu, « littérature postcoloniale » signifie au Japon « littérature qui suit la perte des colonies ", et ne peut être assimilée à ce que l'on appelle communément ainsi ailleurs : il ne s'agit ici pas de la littérature des anciens colonisés, mais des ex-puissances coloniales. Quoi qu'il en soit, l'histoire de la littérature de l'après-guerre comme les recherches portant sur ce domaine ne font quasiment aucune place à cette « littérature qui suit la perte des colonies"; c'est à peine si l'on a regroupé sous l'appellation 《courant des rapatriés» (Gaichi hikiage-ha 外地引揚派) un certain nombre de romanciers dont l'œuvre alimente la littérature de divertissement. De ce courant relèvent des romanciers revenus de Corée après la défaite (Itsuki Hiroyuki 五木寛之 1932-, Kajiyama Toshiyuki 梶山季之 1930-1975), de Chine (Ikushima Jirō 生島治郎 1933-2003), de Mandchourie (Ōyabu Haruhiko 大藪春彦 1935-1996, Uno Kōichirō 宇野 鴻一郎 1934-), et qui font leurs débuts sur la scène littéraire dans les années 1960, en même temps que d'autres auteurs appartenant au courant « des ruines et du marché noir » (Yakeato yamiichi-ha 焼け跡闇市派) comme Nosaka Akiyuki 野坂昭如 (1930-), et 
que la critique journalistique cataloguera comme auteurs d'une nouvelle littérature grand public.

3 N'ont pas été assimilés à ce " courant des rapatriés » les romanciers considérés comme auteurs de « littérature pure » tels Gotō Meisei 後藤明生 (1932-1999), Hino Keizō 日野 啓三 (1929-2002), Kobayashi Masaru 小林勝 (1927-1971), bien qu'ils soient eux-mêmes issus de familles revenues de Corée, de Taiwan ou de Mandchourie, pas plus qu'Abe Kōbō 安部公房 (1924-1993) ou Miki Taku 三木卓(1935-), eux-mêmes revenus de Mandchourie, ou Haniya Yutaka 埴谷雄高 (1909-1997), qui a grandi à Taiwan. En outre, un Akagawa Jirō 赤川次郎 (1948-) ou une Yamamura Misa 山村美紗 (1931-1996), dont les pères étaient de hauts fonctionnaires de l'administration coloniale en Mandchourie et en Corée, et qui, à ce titre, sont profondément liés au système colonial, ne sont pas plus assimilés à ce courant bien qu'ils appartiennent au genre de la littérature de divertissement. Autrement dit, cette dénomination de "rapatriés » de la littérature répond à une définition très restrictive, bien plus étroite par exemple que « courant de l'après-guerre », ou « courant des sans foi ni loi » (Burai-ha 無頼派).

Mais si on adopte une définition plus large de la « littérature postcoloniale » ou de la "littérature des ex-puissances coloniales", on constate que de fait un grand nombre des auteurs les plus importants de la littérature de l'après-guerre sont profondément liés aux anciennes colonies, ou aux territoires occupés militairement. Prenons l'exemple d'Abe Kōbō : lui qui a vécu à Moukden (l'actuelle Shenyang) où son père était professeur à l'école de médecine, n'avait-il pas gardé ancrés dans sa mémoire les vastes paysages et le climat de la Mandchourie ? Son tout premier roman publié en 1948, D'un repère sur un chemin qui s'achève (Owarishi michi no shirube ni 終わりし道の標べに), est imprégné du sentiment de solitude et de la tristesse existentielle qu'éprouve un jeune Japonais lorsque, après la défaite, contraint de regagner une « métropole » qu'il connaît mal et où il doit désormais trouver ses racines, les paysages qui lui étaient familiers de la Mandchourie deviennent soudain ceux d'un pays étranger; et Les fauves se dirigent vers leur pays natal (Kemonotachi wa kokyō o mezasu けものたちは故郷をめざす), publié en 1957, raconte le périple parsemé d'obstacles d'un jeune garçon qui, abandonné en Mandchourie, fuit devant l'avancée des troupes russes pour regagner sa "patrie » du Japon. Or que constate-t-on? Que si les études consacrées à l'œuvre d'Abe se plaisent à aborder la place de la ville dans son imaginaire, le caractère expérimental de son écriture, l'influence du surréalisme, la récurrence de la thématique kafkaïenne, la pensée du désert ou l'irrationalité, on n'y trouve pour ainsi dire aucune analyse concrète de ce qui se trouve au cœur de son expérience, la rémanence du climat mandchourien, la perte du pays natal. Sans doute peut-on expliquer ce manque par le fait que, hormis dans ses romans du tout début ainsi que dans quelques essais et pièces radiophoniques, Abe lui-même ne s'est presque jamais exprimé sur sa Mandchourie, attitude que confortait la tendance générale de la société japonaise de l'après 1945 à passer sous silence l'expérience coloniale.

5 Le héros des Fauves se dirigent vers leur pays natal, roman dans lequel Abe met en scène le plus directement son expérience de la Mandchourie, est un jeune garçon qui tente désespérément de retourner au Japon pour y trouver le pays de ses racines. Mais tandis qu'il fuit la Mandchourie, plus il se rapproche du Japon, plus le Japon de ses racines s'éloigne. Parce que son pays natal, le pays de ses racines est en réalité déjà perdu, révolu, et que son voyage est une errance dans un gigantesque labyrinthe semblable à un désert aride. La femme des sables (Suna no onna 砂の女), où le héros continue 
indéfiniment à creuser son trou dans les dunes, La face d'un autre (Tanin no kao 他人の 顔), histoire d'un homme dans la ville qui a perdu son visage - on pourrait très bien argumenter que tous les principaux romans d'Abe sont fondés sur son expérience de l'après-guerre, l'expérience de celui qui, avec la perte de la Mandchourie, a perdu sa patrie, ses racines, et sa propre identité.

On pourrait dire la même chose de Haniya Yutaka, qui laisse inachevé ce gigantesque roman que sont Les âmes mortes (Shiryō 死霊, 1946-1996), considéré comme l'un des chefs d'œuvre de la littérature de l'après-guerre. Il est tout à fait possible de relier l'expérience qu'il a faite à Taiwan, enfant de la puissance colonisatrice, avec le thème philosophique fondamental chez lui qu'est le "malaise attaché au principe d'identité ", ou à la pensée socialiste qui vise à la réalisation de l'égalité de tous; or il n'existe toujours aucune étude qui s'attache à élucider le sens que revêt dans l'univers de Haniya son " expérience taiwanaise ». Il ne paraîtrait pourtant pas déplacé de lire, dans l'ombre portée des personnages coréens ou chinois qui apparaissent dans Les âmes mortes, une tentative inachevée pour écrire cette expérience de l'Asie dans un Taiwan colonisé.

Si on appelle «littérature des colonies» (Shokuminchi bungaku 植民地文学) les textes littéraires écrits en japonais dans les territoires sous domination coloniale japonaise (péninsule coréenne, Taiwan, Kwantung et Mandchourie, Sakhaline et les îles de Micronésie), on pourrait appeler "littérature post-colonies »(Posuto shokuminchi bungaku ポスト植民地文学) celle d'un ex-pays colonisateur. Et ce terme s'appliquerait à toute la littérature japonaise après 1945 sans exception, de la même manière que « littérature de l'après-guerre » qui s'utilise comme terme générique.

Ōoka Shōhei 大岡昇平 (1909-1988), Takeda Taijun 武田泰淳 (1912-1976), Mishima Yukio 三島由紀夫 (1925-1970), Abe Kōbō, Ōe Kenzaburō 大江健三郎 (1935-), Nakagami Kenji 中上健次 (1946-1992), Murakami Haruki 村上春樹 (1949-) : parmi ces grands noms de l'histoire littéraire de l'après-guerre, rares sont ceux dont aucune œuvre n'entretient de rapports avec les anciennes colonies ou territoires occcupés. Et il est particulièrement intéressant de noter que deux de ces auteurs, Nakagami Kenji et Murakami Haruki, nés après 1945, ont tous deux écrit de longs romans dans lesquels il est question de la Mandchourie.

Quand une mort trop tôt venue l'a cueilli, Nakagami Kenji travaillait à un roman resté inachevé, Tribu étrangère (Izoku 異族). Tribu étrangère est l'histoire d'hommes qui, liés par une sorte de relation de fratrie du fait qu'ils portent sur la poitrine une tache en forme de Mandchoukouo, sont amenés à se retrouver comme dans le roman Histoire des huit chiens de Satomi (Nansō Satomi Hakkenden 南総里見八犬伝) de Kyokutei Bakin 曲亭, 馬琴 (1767-1848) ${ }^{1}$. Le héros est issu d'une famille de discriminés, les autres sont coréen, aïnou, uchinanchu (d'Okinawa), noir américain résidant au Japon, et tous constituent la tribu étrangère. Tribu qui offre donc en somme un assemblage de toutes les minorités du Japon, et si on considère qu'Okinawa et Hokkaidō sont des colonies intérieures, on peut comprendre ces personnages comme étant issus des colonies situées aux marges de la puissance coloniale qu'est le Japon. On peut donc supposer que le dessein de l'auteur était de décrire le présent d'un Japon " post-colonies » à travers les révoltes et les rébellions de ceux qui viennent de ces anciennes colonies. Le roman s'interrompt au 
moment où le héros et les principaux personnages partent pour les Philippines, région jadis désignée au Japon comme Pacifique Sud Extérieur (Soto nan'yō) et occupée militairement au début de la Guerre du Pacifique. L'armée japonaise s'est emparée de cette colonie américaine sous prétexte de la libérer, ce qui lui a permis de prendre la place et d'accaparer les droits de la puissance coloniale.

Ōoka Shōhei, mobilisé juste avant la défaite sur le front des Philippines et fait prisonnier par les troupes américaines, a par la suite écrit Journal d'un prisonnier de guerre (Furyo-ki 俘虜記, 1948) ou Chroniques de la bataille de Leyte (Reite senki レイテ戦記, 1972) dans le prolongement de son expérience. Mais il reconnaît en un retour autocritique sur son œuvre que, s'il a écrit sur les combats opposant les Japonais et les Américains, il n'a pas su accorder l'attention qu'ils méritaient aux premières des victimes, les Philippins. Cette anecdote est très révélatrice du fait que dans la littérature de l'après-guerre ont été négligés, voire oubliés les habitants des colonies et des territoires occupés. Nakagami Kenji n'a-t-il pas tenté à travers son œuvre de faire jouer aux Philippines un rôle symbolique pour compenser cette absence de la colonie dans la littérature ? C'est probablement en lien avec la situation postcoloniale telle qu'il la percevait qu'il s'intéressait autant à la Mandchourie, à la péninsule coréenne, aux archipels du Pacifique.

11 Murakami Haruki est généralement considéré comme le porte-drapeau de la littérature post-après-guerre et comme un romancier représentatif de la période post-moderne. Qu'il se soit référé largement, dans la plus longue et sans doute la plus importante de ses œuvres, La chronique de l'oiseau à ressort (Nejimakidori kuronikuru $\mho_{2}$ じまき鳥クロニ クル, 1992-1995), à un épisode historique concernant le Mandchoukouo, pays satellite $\mathrm{du}$ Japon, montre sans doute que la littérature post-après-guerre est également une littérature post-colonies.

12 Le Mandchoukouo, érigé par le Japon en violation des recommandations de la SDN, avait une frontière commune au nord avec l'URSS. On désigne sous le nom d'Incident de Nomonhan les combats qui opposèrent l'armée soviéto-mongole et l'armée japonaise et du Mandchoukouo sur cette frontière. Dans La chronique de l'oiseau à ressort est relatée, par le Lieutenant Mamiya, une affaire d'espionnage nippo-russe qui a trait à Nomonhan. Un espion militaire japonais, capturé au-delà des frontières par les Mongols, est sauvagement torturé.

13 Pourquoi Murakami, qui est d'une génération n'ayant aucune expérience directe ni de la Mandchourie ni même de la guerre, a-t-il intégré dans son univers romanesque ces expériences appartenant à la génération de ses parents ? Déjà, dans La course au mouton sauvage (Hitsuji o meguru bōken 羊をめぐる冒険, 1982), il était question d'un mouton qui porte une marque en forme d'étoile et qui se trouvait en Mandchourie; dans La chronique de l'oiseau à ressort, est évoqué par exemple, en plus de l'affaire de Nomonhan, le massacre des animaux du zoo de Xinjing (l'actuel Changchun): les personnages mentionnent ainsi l'expérience coloniale réelle qui a pour cadre la Mandchourie, et ces récits viennent se superposer aux récits concernant le monde d'aujourd'hui, induisant une stratification en de multiples couches de l'univers romanesque.

14 L'incident de Nomonhan avait pour origine une question de frontières : Murakami n'y aurait-il pas vu la preuve de l'inanité attachée au fait de tracer une ligne artificielle et 
de séparer un en deçà et un au-delà de la ligne dans ces espaces vides, ces steppes où il n'y a rien? À moins que la signification de cet épisode ne se cache dans le fait de transgresser la ligne pour passer d'un en deçà à un au-delà, puis d'un au-delà à un audeçà.

Quoi qu'il en soit, il semble bien que l'on puisse trouver ici un sentiment de méfiance et de rejet à l'égard des frontières et des démarcations. Bien entendu, il existe des lignes de séparation, des frontières invisibles et indéniables entre puissances coloniales et colonies, dominants et dominés. Est-il possible de franchir ces lignes? C'est cette question d'actualité que poserait ce roman.

Dans 1Q84, son dernier roman, Murakami Haruki fait lire à Aomame, l'un de ses personnages principaux, un livre sur les chemins de fer de Mandchourie, à un autre, Tengo, un livre d'Anton Tchekhov, Voyage à Sakhaline. Sakhaline (Karafuto en japonais), était une colonie japonaise (jusqu'au $50^{\mathrm{e}}$ degré de latitude nord), et la mention de l'ouvrage de Tchekhov, ou encore l'apparition de Tamaru, un personnage fils de Coréen de Karafuto replié au Japon, suggère que ce roman entretient des liens profonds avec Sakhaline en tant que colonie japonaise. Comme Tchekhov lui-même, Murakami éprouverait une passion indiscutable, qui déclencherait cette "fièvre de Sakhaline" qu'il ne comprendrait pas bien lui-même. La Mandchourie, Karafuto, tous ces vides sur la carte de la périphérie du Japon par lesquels Murakami Haruki est attiré, ne matérialisent-ils pas sa volonté inconsciente de régler son compte à l'histoire moderne $\mathrm{du}$ Japon comme expression du colonialisme?

Le dernier roman en date de Tsushima Yūko 津島佑子 (1947-), Barbare, si barbare (Yabanna，amarinimo yabanna 野蛮な、あまりにも野蛮な，2009), est une œuvre ambitieuse que l'on doit à une auteure parmi les plus actives aujourd'hui au Japon. Comme La chronique de l'oiseau à ressort, ce roman est constitué de plusieurs strates, l'une qui porte sur le Japon de l'avant-guerre et de la guerre, l'autre sur le Japon d'aujourd'hui, avec une superposition de récits portant sur le Taiwan de l'époque coloniale (1895-1945) et sur les années 2000. Le dessein de Tsushima ici est de renverser le couple d'opposition barbarie-civilisation, et de substituer l'un à l'autre. Les populations indigènes de Taiwan étaient considérées dans les classifications japonaises d'alors comme l'une des trois grandes peuplades primitives (les deux autres étant les aïnous d'une part, et les "indigènes du Pacifique Sud» d'autre part). Ainsi, plus barbares que ces indigènes aux coutumes de coupeurs de tête, n'y avait-il pas le Japon et les Japonais qui, depuis Meiji, s'étaient efforcés de construire un pays civilisé, rivalisant avec les plus grandes puissances mondiales, et s'enorgueillissaient d'avoir atteint cet objectif? Un postmodernisme permettant de mettre en perspective le Japon moderne ferait de l'empire colonial un sujet essentiel en études littéraires. C'est un rite de passage incontournable pour construire une littérature qui soit, véritablement, celle de l'après-après- 1945. 


\section{NOTES}

1. Voir à propos de cette œuvre l'article de Fanny Bertu, « Kyokutei Bakin, un précurseur en matière de recherche littéraire ", dans le numéro 16 de Cipango. 